\title{
Computer-based, automatic recording and illustration of complex archaeological artifacts
}

\author{
Ayelet Gilboa ${ }^{\mathrm{a}, *}$, Ayellet Tal ${ }^{\mathrm{b}}$, Ilan Shimshonic, Michael Kolomenkin ${ }^{\mathrm{b}}$ \\ ${ }^{a}$ Dept. of Archaeology, University of Haifa, Haifa, Israel \\ ${ }^{b}$ Dept. of Electrical Engineering, Technion, Haifa, Israel \\ ${ }^{c}$ Dept. of Information Systems, University of Haifa, Haifa, Israel
}

\begin{abstract}
We report on the development of a computerized automatic system to illustrate complex archaeological objects. The illustrations are based on 3D scans of the artifacts. The 3D models can be automatically translated, by new algorithms specifically designed for this purpose, into $3 \mathrm{D}$ or $2 \mathrm{D}$ line drawings; into colored images that emphasize the salient shape attributes of the artifacts and of the 3D designs on them; and to images that enhance faint/eroded designs that are otherwise difficult to discern. These illustrations are intended to replace traditional, manual drawings, which are very expensive to produce and not accurate enough. Our illustrations also provide a better visualization tool than the 3D models themselves. Though $3 \mathrm{D}$ scanning already improves the visibility of objects and their features, it does not suffice for rapid visual recognition. Our system generates efficient, objective, accurate and simplified representations of complex objects and the designs on them from any number of required views.
\end{abstract}

Keywords:

Archaeological drawing, computerized illustrations, 3D technology in archaeology, non-photorealistic rendering

\footnotetext{
* Corresponding author

Email addresses: agilboa@research.haifa.ac.il (Ayelet Gilboa), ayellet@ee.technion.ac.il (Ayellet Tal), ishimshoni@mis.haifa.ac.il (Ilan Shimshoni), michkol@tx.technion.ac.il (Michael Kolomenkin)
} 


\section{Introduction}

This paper is part of a world-wide effort to overcome the impasse created by current procedures of recording/illustrating archaeological artifacts. Traditionally these were, and to a large extent still are, manual procedures, which, on the one hand, are not accurate enough, prone to biases, and very selective, and on the other, extremely costly and time consuming (see below). Indeed, as in other aspects of archaeology, the need for a computerized revolution has been recognized and the realization that $3 \mathrm{D}$ imaging of archaeological artifacts is essential for cultural resource management and for archaeological research is quickly spreading. Several groups around the globe are experimenting with the implementation of existing technologies or developing new ones to this end. 3D scans of objects have, for example, been used for translating line drawings in archaeological publications into 3D images; calculating capacities and symmetry of (usually axially-symmetric) vessels, and virtual restoration of broken/fragmentary ceramic pots and other objects. Chiefly, however, 3D technology is used to store, visualize and disseminate the entire geometric and textural information of objects for scholarly purposes, educational projects and culture resource management. Some examples out of many for all the above are the Virtual Hampson Museum, Arkansas (Virtual Hampson, 2010); various large scale projects such as the MURALE project at Sagalassos (Van Gool et al., 2000); the Telamon Temple Project (Brutto and Spera, 2011); the 3Dk project at the PRISM lab, University of Arizona (PRISM, 1997); and the various projects of the S.H.A.P.E. center at Brown University (S.H.A.P.E, 2002). Other such studies are presented in Aliaga et al. (2008); Hanke et al. (2008); Levoy et al. (2000); Li et al. (2011); Martínez Carrillo et al. (2008); Pires et al. (2006); Simon et al. (2002); Toler-Franklin et al. (2010). Recently, computerized means have also been developed for the computerized comparison/classification of plain ceramics with axial symmetry (e.g., Adler et al., 2001; Hörr et al., 2007; Kampel and Sablatnig, 2007; Karasik and Smilansky, 2011); for the latter see Section 2.3 below.

This paper - culminating from our technical research in the last few yearsis concerned with the visualization of complex archaeological objects. Our previous results were presented at computer-technology venues, where we described in detail the solutions to the specific algorithmic problems involved. This paper has two main goals: (1) To present a complete description of the technology, its rationale and its epistemological advantages for supporting 
archaeological research and cultural resource management. (2) To provide archaeologists with an operative system using this technology.

The system presented here uses 3D scans of archaeological objects in order to produce images that visualize them better than the 3D models themselves. The main goal is to facilitate archaeological research, where comparison of objects and the designs on them to other relevant 'similar' objects is a mandatory first step. Toward this end scholars are oftentimes required to browse through illustrations of hundreds, oftentimes thousands of objects, still mostly on paper - a truly Sisyphean task. To do this they require images which-beyond being accurate - must enable a quick recognition of the artifact's idiosyncratic shape/design attributes.

\section{An overview of previous and current recording/publication meth- ods of complex archaeological objects}

\subsection{Traditional methods: manual drawings and photographs}

In any but the smallest excavations, several categories of objects, mainly potsherds and other ceramic objects (in 'historical' periods), or flint tools (in prehistoric ones), are found in thousands, oftentimes, in large-scale excavations - millions. Traditionally, in order to record and publish these objects, the archaeologist proceeds in the following manner.

Some categories of objects are recorded/published as 2D photographs only (for which see further below in this section). Equally frequently, artifacts in site reports, other archaeological studies and, for example, in museum catalogues - are documented and published by meticulous 2D line drawings (Fig. 1:1, 3, 4) or by non-photo-realistic renderings by stippling (Fig. 1:2, $5,6)$. These are produced manually by trained artists. The illustrations reproduce the object's external shape, decoration, and usually also its inner structure (e.g., the thickness of its fabric), by providing one or more sections through it. The styles of these drawings vary greatly between publications, and oftentimes within publications, since they reflect the different artists' hands.

Due to the large numbers of artifacts and the time-consuming process of manual drawing, the documentation and ensuing analysis of some categories of objects are the main cause of what is often termed "archaeology's dirty secret" - the severe delay, sometimes by decades, in publishing site reports. From our own experience it takes a competent artist anywhere between an hour and a day and occasionally more to draw fragments/objects such as 
those illustrated in this paper, depending on size and complexity of the designs. To this should be added the time spent by the archaeologist 'guiding' the drawing process, negotiating corrections and finally approving the drawing.
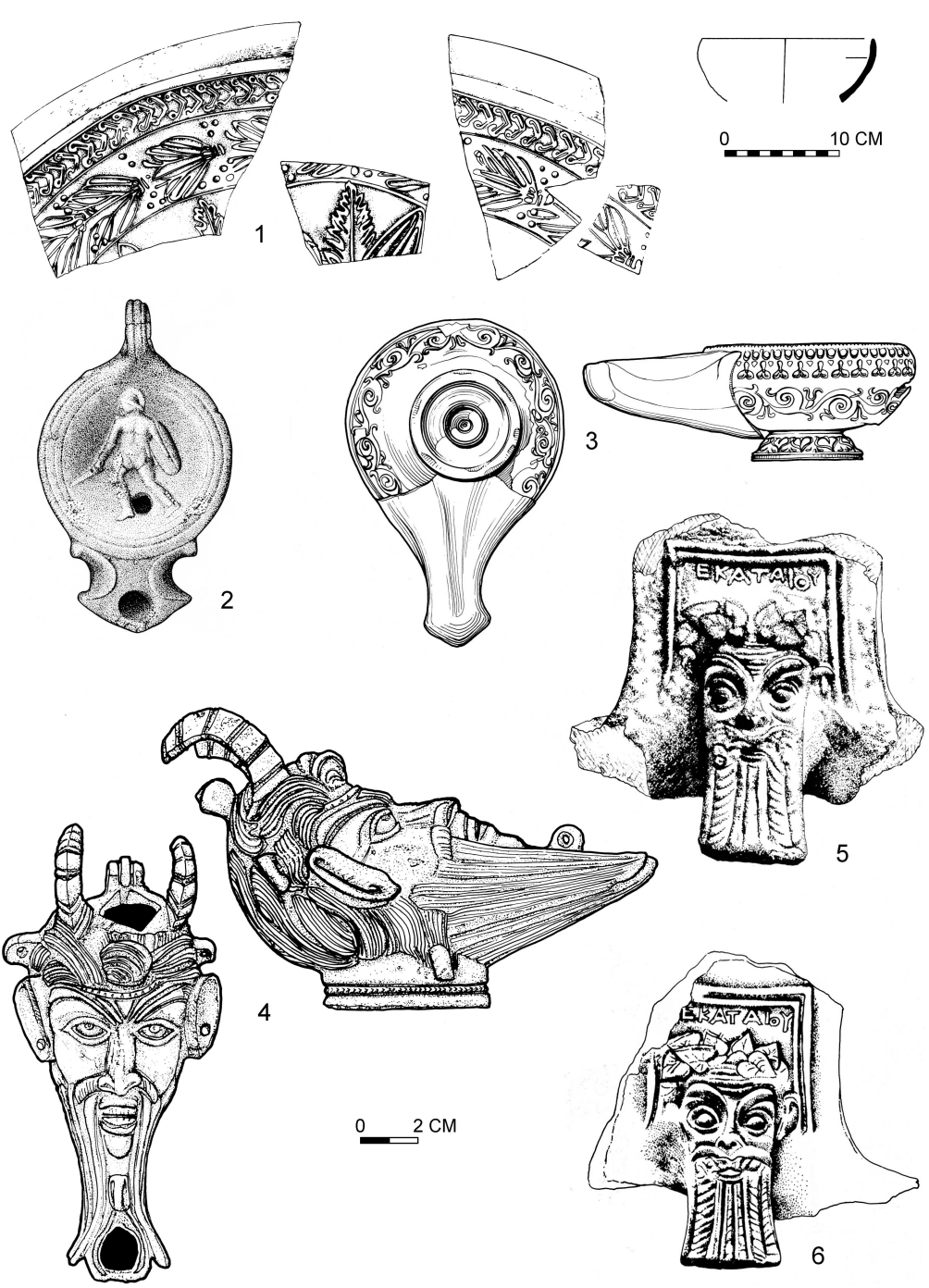

Figure 1: Examples of manual drawings of complex objects. (1) relief bowl (RosenthalHeginbottom, 1995, Fig. 5.3:9); (2) oil lamp (Goethert-Polaschek, 1985, Pl. 3:17e); (3, 4) oil lamps (Bailey, 1996, nos. Q3721, Q3575); (5, 6) brazier fragments (RosenthalHeginbottom, 1995, Figs. 5.1:3, 4).

In the archaeological literature, the merits and demerits of such drawings 
(also versus photographs) are usually discussed very succinctly (Adkins and Adkins, 1989; Dillon, 1992; Gooding, 2008; Griffiths et al., 1990; Joukowsky, 1986; Steiner, 2006). However, the very fact that this costly method is so common in portraying complex objects is testimony to its usefulness. Indeed, these drawings, especially line-drawings, are very user-friendly since, at a quick glance, they convey and highlight all the pertinent morphological information regarding the objects and their decorations/surface treatment (or rather the information considered pertinent; see more on this in Section 2.2).

Some examples of publications featuring dozens to several hundreds of manual drawings of complex objects are the following. Lamps with relief decoration, for example, are represented by two line drawings each in Rosenthal-Heginbottom (1995, figs. 5.15-5.24). Hundreds of drawings in different styles, with one, two or more views per object, accompanied by a lesser quantity of photographs appear in Bailey (1996, pls. 1-108). Numerous manual drawings in different styles were published in Gassner (1997, pls. 1-9, 14-20, 63-67). About 200 relief-decorated lamps published mainly by line drawings, usually with two views per lamp, appear in Mlynarczyk (1997, figs. 3-195).

Beyond costs, however, there are more drawbacks to this illustration method: (1) Manual drawings reflect the artist's proficiency, the archaeologist's often tacit preconceptions (and the patience of both) and are prone to inaccuracies and biases (Gooding $(2008,17)$; see also discussions in Orton et al. (1993); Pobelome et al. (1997); Mara and Sablatnig (2005)). Can it really be stated that the drawings in Fig. 1 are accurate renderings of the shape and designs of the objects? Another critical issue, of course, is that the users are unable to assess the accuracy of the drawings. (2) Furthermore, due to time, space and budget constraints usually only one, and only seldom more views of the objects are illustrated in publications. The result is that not all the morphological information is recorded. With ever-shrinking budgets a second outcome is that excavation projects, for example, tend to be more selective with the publication of such objects than they would have, had there been a more efficient means of illustration. All these problems are, of course, significantly aggravated the more complex the objects are.

In many site reports (and other publications), the financial problems have been 'solved' simply by forgoing the line drawings - and publishing complex objects by photographs only, with black-and-white $2 \mathrm{D}$ photos still being the norm. Again, usually one view of the object is provided, other than for very 'special' items. Examples for the various 'selections' in publications 
follow. In Howland (1958) there are photographs only and only one view of each object (pls. 1-21). In Perlzweig (1961) lamps are published mostly by photographs of one view, with few line-drawings illustrating the objects' sections, and in pl. 51 also designs on a few selected lamps. In Rotroff (1982) about 400 objects with relief decorations were published by photographs in pls. $1-70$, and only about 70 were illustrated by line drawings as wellpls. 73-97. In Goethert-Polaschek (1985) most of the objects were published by photographs of one view, in pls. 16-77, and only representative types per period also illustrated by line drawings in pls. 1-15; and cf. a similar selection in Mandel (1988) with about 50 objects illustrated by meticulous line drawings, Typentafeln I-VI.

Photographs, however, are not a satisfactory solution for other reasons as well. Artifacts are often worn and their shape and/or design are not clearly-enough discerned through the photograph. Another factor obscuring designs on such objects is the differential preservation or appearance of the surface of the object (texture and color), caused by post-depositional and other processes. When actually looking at an object, it is usually possible to distinguish, for example, between real engraved marks on seals, inscribed tablets etc., and various scratches caused by use, the deposition environment and modern careless handling. This distinction, however, is often obfuscated in photographs. Thus, both from our own and others' experience photographs are just not enough, especially when artifacts with detailed and delicate/intricate designs are concerned. Surely, the situation is rapidly improving, with higher-quality color photographs replacing the black-and-white ones. With reduced printing costs these photos will figure more prominently in publications, and of course completely replace the black-and-white ones when site reports etc. (or at least their digital images) are published electronically. Similarly to the drawings, however, photographs cannot store and disseminate the entire morphological information about the object.

\section{2. $3 D$ scans}

As shortly discussed in the introduction, archaeological and other cultural heritage projects are increasingly moving on to recording objects by various 3D technologies, which to a large extent solve all the problems enumerated above. 3D images are accurate, simple and relative quick to generate and they retain all the information regarding the objects. The main problem with such $3 \mathrm{D}$ models is that even when published electronically they do not convey the pertinent morphological structure in a manner that, similar to the manual 
drawings discussed above, enables rapid and effortless visual recognition. This drawback becomes a severe one especially when such objects must be viewed en masse, as in the case of comparative artifact research.

We therefore seek technologies that will improve on the scans by creating means of imagery that have the same advantages as the traditional manual drawings. Such technologies are termed non-photorealistic rendering.

As noted by Gooch and Gooch (2001) non-photorealistic rendering brings art and science together, concentrating less on the process and more on the communication content of an image. Techniques that have long been used by artists can be applied to computer graphics to emphasize subtle attributes and to omit extraneous information (for the general role of art in science see also Gooding, 2008). Moreover, psychological scholarship has demonstrated the visual advantage of line drawings over realistic shape rendering. For example, Biederman and Ju (1988) and Biederman (1995) demonstrated the efficiency of edge-based (or contour) representations, i.e. line drawings, over surface rendering for providing real-time recognition of the morphology of objects. The line drawings were shown to be effective especially in cases where texture and color were not the important diagnostic features of the objects and when individuals were exposed to the images for short episodes.

The methods we present here are therefore intended to combine the comprehensiveness, accuracy and efficiency of 3D scans with the eye-friendliness and the information conveyed by the non-photorealistic renderings of the models.

\subsection{Computer-generated line-drawings on $3 D$ objects}

Very few groups have developed to date algorithms designed to generate automatic drawings of archaeological artifacts and hardly any have produced operative tools that can be used by archaeologists. Recently, a genuine breakthrough towards automatization of the entire procedure has been reported by Karasik and Smilansky (2008), with references to works of other groups. They present a method of computerized recording and drawing of profiles of potsherds belonging to plain vessels with axial symmetry (and of complete such vessels). The shape of the potsherds/vessels is recorded with a structured-light scanning system that captures the entire shape of the objects in an accurate and efficient manner. These images can then be 'translated' automatically into 2D line drawings of profiles, reproducible on paper. This is done by specially-developed algorithms which compute the items' rotational 
axis and thus align them correctly, and subsequently compute the mean profile of the potsherds/vessels. This mode of recording/drawing plain pottery is quickly spreading in Israel and also beyond (e.g., Levy et al., 2010, 146-147).

Beyond the automatic illustration of pottery, efficient computerized typology and classification of plain pottery has been achieved using these means (Gilboa et al., 2004; Karasik and Smilansky, 2011); for advances regarding the computerized representation and analysis of lithics, see for example, Grosman et al. (2008).

Plain, axially symmetric pottery, however, accounts of course for only part of the artifactual assemblages we excavate. The next step is to develop means to achieve similar results with objects whose shape is more complex and/or which bear designs and inscriptions.

\section{Producing line drawings of complex objects}

Complex objects such as coins, figurines, oil lamps, stamps/seals, etc. have idiosyncratic shape attributes, and they require specific methods of documentation and classification. A differentiation should be made between two major categories: those where all the pertinent information (shape and design) is three dimensional and those that also bear painted designs (such as painted ceramics). Mathematically, these two categories present totally different challenges. In the first, the geometry is complex and the challenge is to translate the human intuition used in manual object illustration into 3D geometric shape analysis. Regarding objects of the second category there is another challenge, which lies within the realm of image processing of faded-painted objects. In this paper we are only concerned with the former category.

\subsection{Related Work}

The work presented here is at the crossroads of three fields of nonphotorealistic rendering: line drawing, coloring (gray-scale depiction), and shape enhancement.

\subsubsection{Line Drawing}

Lines on 3D objects can be classified as view-dependent or view-independent curves. View-dependent curves depend not only on the differential geometric properties of the surface, but also on the viewing direction. They change whenever the camera changes its position or orientation (DeCarlo 
et al., 2003; DeCarlo and Rusinkiewicz, 2007; Judd et al., 2007; Koenderink, 1984). Therefore they can be utilized for visualization only. Conversely, view-independent curves do not change with respect to the viewing direction, and hence can also be used for shape analysis. We therefore focus on the latter.

Many of the existing algorithms for line-drawing detect ridges and valleys, which are the extrema of principal curvatures (Luo et al., 2011; Ohtake et al., 2004). Other types of curves are parabolic curves, which partition the surface into hyperbolic and elliptic regions, and zero-mean curvature curves, which classify sub-surfaces into concave and convex shapes (Koenderink, 1984). They correspond to the zeros of the Gaussian and mean curvature, respectively. Finally, Demarcating Curves are the zero-crossings of the curvature in the curvature gradient direction (Kolomenkin et al., 2008). While portraying important object properties, all the aforementioned curves sometimes fail to capture relevant features, such as weak edges, highly curved edges, and noisy surfaces.

\subsubsection{Coloring}

A different visualization technique attempts to give a color value to every point on the surface. For instance, the Polynomial Texture Maps system (PTM) allows the user to change the lighting direction interactively, to help visualize the object 'naturally', in order to enhance surface details that may otherwise be difficult to see. Other methods propose to artificially 'color' each point on the surface, emphasizing local geometry properties. For instance, Kindlmann et al. (2003) and Kolomenkin et al. (2008) suggested coloring the surfaces using mean-curvature shading, where the color is a function of the mean curvature of the point. In Rusinkiewicz et al. (2006) exaggerated shading is proposed, which is based on dynamically adjusting the effective light position for different areas of the surface. In addition, algorithms have been developed to deal with specific types of archeological objects. An example of such an algorithm was presented by Mara et al. (2010). The algorithm extracts characters from 3D scans of cuneiform tablets, using a shading method they developed, which is similar to mean-curvature shading.

\subsubsection{Shape enhancement}

Finally, differential geometry techniques can be utilized to enhance 3D features and eliminate noise. This is done using adaptive filtering algorithms, which smooth (or denoise) the surface, while keeping the features intact or 
enhancing them (Eigensatz et al., 2008; Fleishman et al., 2003; Kolomenkin et al., 2011; Yoshizawa et al., 2006). The techniques differ in the criteria (cost function) they attempt to satisfy (minimize).

In this paper, we introduce a system that provides an integrated solution for the problems described above, regarding line-drawing, coloring, and feature enhancement. The user may choose any combination that best helps visualize the archaeological artifacts.

\subsection{The D.o.R (Drawing over Reliefs) system}

As already mentioned, to produce a clear drawing of the artifacts we propose three types of visualizations: 3D line drawings, coloring of 3D objects, and enhancement of the object's features that may have eroded over time (Fig. 2). We elaborate below.

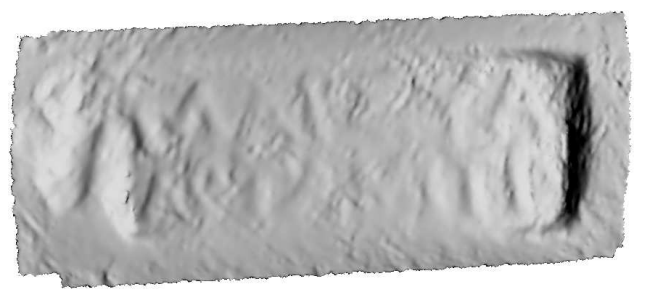

3D model

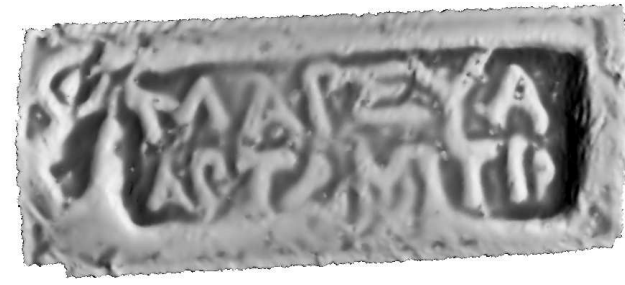

Coloring

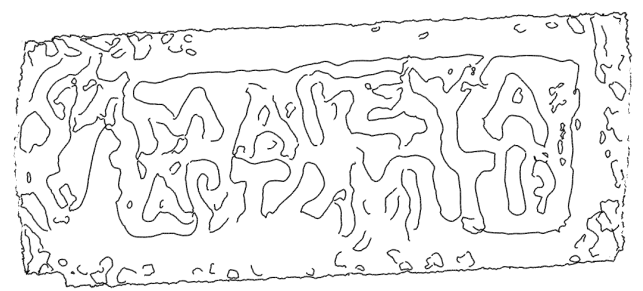

Line drawing

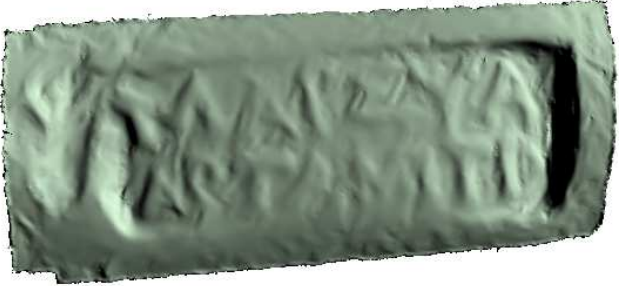

Enhancement

Figure 2: Examples of different visualization options of the D.o.R system (a worn Hellenistic stamped handle).

\subsubsection{Input}

The system is based on 3D models that can be generated by any 3D acquisition technique. For the relatively small objects reproduced in this paper we used a structured-light 3D scanning system produced by Polygon 
Technology, Darmstadt, Germany. Other than the projector it comprises of three digital cameras: two record the objects' geometry and one records their texture/color (the latter can be operated at will, and has not been used for the procedure described here). This is the same system described and illustrated in Karasik and Smilansky (2008). The readers are referred to that publication for a detailed description and illustrations of the mode of operation of the system's hardware and software (the QTSculptor program), and the mode of interaction between the individual performing the scanning and the machine. For the small objects presented here the density of points on the surface of the scanned image was on the average $0.1 \mathrm{~mm}$ or less.

As opposed, however, to the protocol for the plain potsherds described by Karasik and Smilansky (2008), we did not attempt to scan several objects simultaneously, since for the complex objects we are dealing with here we aimed at the highest possible scanning resolution. This means that the pace of scanning was slower than that reported by Karasik and Smilansky. Typically, for objects that were scanned only partially (such as the stamped handles in figs. 2,10$)$, or that could be completely viewed by the cameras when they were turning on the turntable (such as the fragments of relief bowls in figs. 7,8$)$, about 10 minutes were required to complete the scanning and registration. For larger objects scanned in the round (figs. 3, 5, 6, 9) scanning time per item was about double (20 minutes, occasionally more). The reason is that in order to capture the entire shape of these objects, they had to be placed on the rotation table (or suspended from a frame, as described by Karasik and Smilansky (2008)) in at least two different positions. The resulting image (e.g. fig. 5 left; see the procedure described in Karasik and Smilansky $(2008,1150-1151)$ is an accurate 3D image of the object's geometry. This 3D representation is a VRML file that defines the boundary surface as a collection of triangles in space. Usually, there are 100,000-200,000 triangles per model. The VRML file can be converted to several other 3D formats; we for example also use PLY.

\subsubsection{D line drawing}

Given a surface in 3D, the goal is to draw on it lines (curves), which convey its 3D features (Fig. 3 right). In particular, we aim to develop a mathematical formalism yielding results that imitate manual archaeological draftsmanship. Such curves can either be used in themselves for drawing purposes, or as advocated below, as a basis for coloring algorithms, described in the next section. 


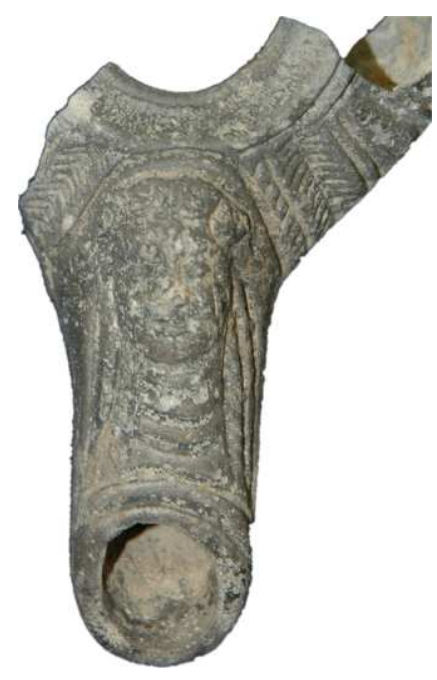

Photograph

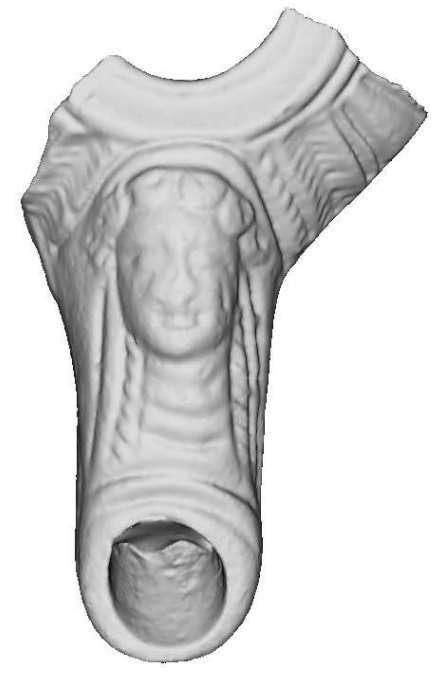

3D model

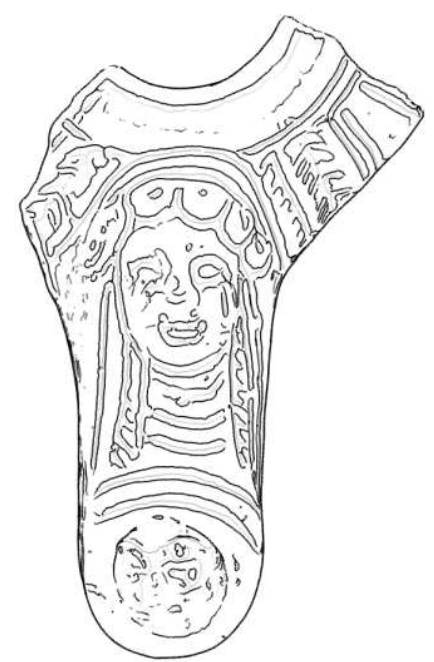

Line drawing

Figure 3: Photograph, 3D model and a line drawing of a Hellenistic lamp fragment

Specifically, given a surface, we can imagine it locally as a terrain with ridges and valleys. Intuitively, the lines drawn should run over the slopes between the ridges (the convex sub-surfaces) and the valleys (the concave sub-surfaces). In other words, the curves should be the loci of the 'strongest' inflections on the surface (i.e., where the transition from convex to concave is the fastest). The challenge is to find the curve points and the direction along which we search for these inflections (Kolomenkin et al., 2008, 2009).

One way to address this problem is to consider a surface as an unknown smooth manifold (base), on top of which a local height function is defined (e.g., a relief). The function can be considered locally as a standard image defined on the tangent plane of the base. Our edges are the edges of this local image, i.e., a surface point $p$ is a relief edge point if it is an edge point of this image. The problem is that the base surface is unknown.

Briefly, our edges (so called Relief Edges) are defined as the zero crossings of the normal curvature in the direction perpendicular to the edge. Initially, the edge direction is estimated for every point by fitting a step edge model to the surface. Given the edge directions, the precise edge localization is obtained. Figure 4 illustrates the surface model.

Formally, given a surface $S(u, v): \mathbb{R}^{2} \rightarrow \mathbb{R}^{3}$, we assume that it consists of a smooth base surface $B(u, v): \mathbb{R}^{2} \rightarrow \mathbb{R}^{3}$, and a function (local image) 


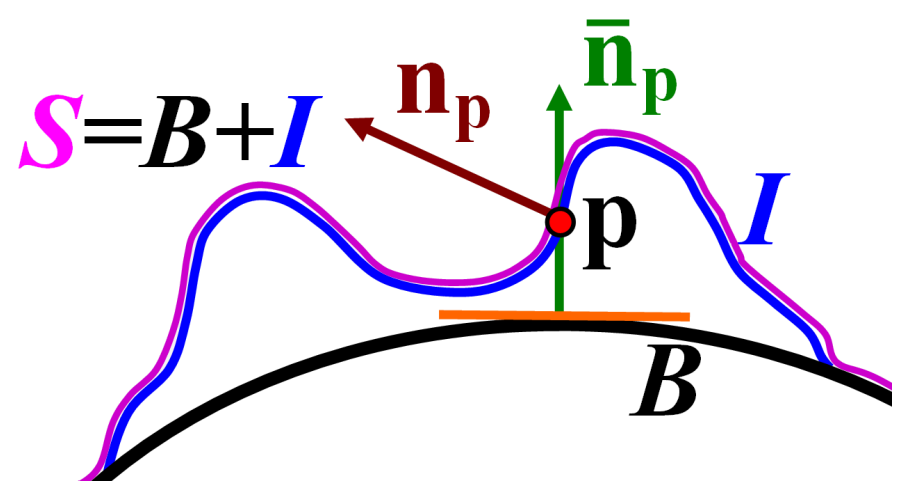

Figure 4: Computing Relief Edges: The surface $S$ (magenta) is composed of a smooth base $B$ (black) and a function $I$ (blue). Function $I$ at point $\mathbf{p}$ can be locally viewed as an image defined on the tangent plane (orange) of the base. Point $\mathbf{p}$ is a relief edge point if it is an edge point of this image. The normal $\mathbf{n}_{\mathbf{p}}$ (brown) is the normal of $S$ and $\overline{\mathbf{n}}_{\mathbf{p}}$ (green) is the normal of $B$ corresponding to $\mathbf{p}$.

$I(u, v): \mathbb{R}^{2} \rightarrow \mathbb{R}$, defined on $B:$

$$
S(u, v)=B(u, v)+\bar{n}(u, v) I(u, v)
$$

where $u$ and $v$ are the coordinates of a planar parametrization and $\bar{n}(u, v)$ : $\mathbb{R}^{2} \rightarrow S^{2}$ is the normal of $B$ on the unit sphere. We assume that $B$ is locally a manifold and that its curvature has a smaller value than the curvature of $I$. The decoupling of $S$ into $B$ and $I$ is unknown. Note that in the special case of an image, $B$ is the image plane, $\bar{n}(u, v)$ is constant and $I$ is the image intensity.

The goal is to detect edges on $S$ that correspond to edges on the local images $I$. We consider the common definition of edges in images as points at which the derivative obtains a maximum in the gradient direction. We will show that the edges can be detected without accurately estimating $B$ or its normal $\bar{n}$ - a rough estimate suffices.

Relief Edges are computed in two steps: estimating the edge direction at every point and determining the relief edge points using this estimation. We elaborate on these steps below.

The edge direction $\theta$ and its magnitude $\alpha$ are estimated by fitting an edge model that best approximates the surface locally. In other words, we seek $(\hat{\theta}, \hat{\alpha})$ that minimize the difference between the edge model $E(\hat{\theta}, \hat{\alpha})$ and the 
local surface model $S$. We define the approximation error as:

$$
\operatorname{Err}(\theta, \alpha)=\int\|E(\theta, \alpha)-S\|^{2} \rho d \rho d \phi,
$$

where the integral is defined over a neighborhood of $p$ and $\rho$ is the Jacobian of the polar coordinates substitution. The optimal edge is determined by

$$
(\hat{\theta}, \hat{\alpha})=\arg \min \operatorname{Err}(\theta, \alpha) .
$$

We reformulate (2) in terms of vectors in the polynomial space of $\cos \theta$. and $\sin \theta$. This formulation allows us to represent our optimization problem as the problem of finding the roots of a third-order polynomial of $\sin ^{2} \theta$.

The previous step computed $\hat{\theta}$ and $\hat{\alpha}$ for every point on the surface. Our goal is to find the edge points, which are the loci of points where the gradient obtains maximum in the gradient direction.

For a smoothed step edge the gradient direction is perpendicular to the edge direction. Therefore, the loci of the relief edges are the zero crossings of the curvature in the direction perpendicular to the edge direction $\hat{\theta}$.

We can now formally define a relief edge point. Let $g_{p}=[\cos \hat{\theta}, \sin \hat{\theta}]$ be a vector perpendicular to the edge direction at point $p$, and let

$$
G_{p} \stackrel{\text { def }}{=} g_{p}^{T} I I g_{p}
$$

be the value of the normal curvature in the gradient direction at $p$, where the symmetric matrix II is the second fundamental form (Do Carmo, 1976).

Definition: Point $p$ is a relief edge point if $G_{p}=0$.

As can be seen in Figures 2, 3, the line drawings produced by the system are not clear enough to consist, in themselves, a good enough representation of the object (for which see below, coloring). However, they are pertinent for two main reasons. (1) They visualize the graphic output of the algorithm. (2) As discussed further below, they are useful when combined with other visualization methods.

\subsubsection{Coloring of $3 D$ objects}

This is a method for artificially coloring objects. The key idea is to give a gray-scale value to each point on the surface, according to its normal curvature in a specific direction. This increases the contrast of the features, 


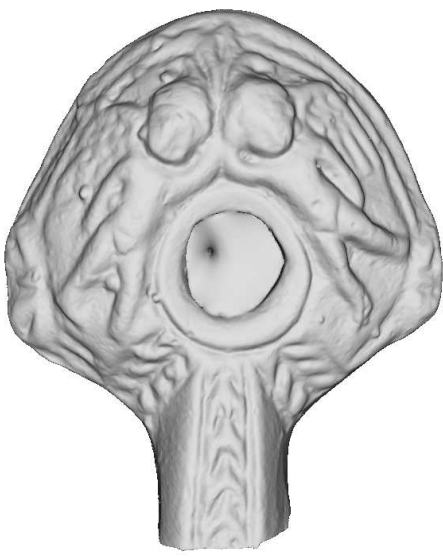

Model

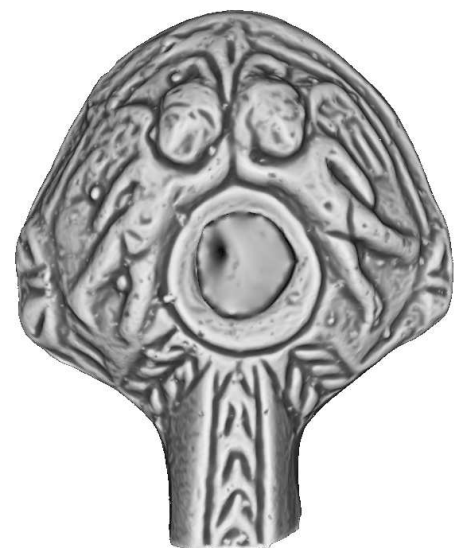

Colored

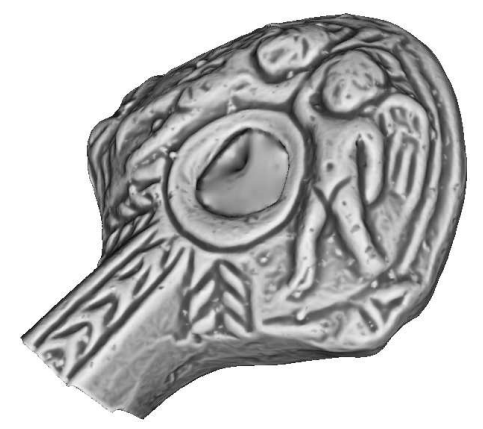

Another view

Figure 5: Coloring (a Hellenistic oil lamp)

thus enhancing them. The challenge is to define this prominent direction (termed the prominent field) everywhere on the surface, and compute it.

Recall our definition of relief edges, which are the loci of the strongest inflections. If we follow the direction along which we obtain the strongest inflection, we will move in the fastest possible way from convex regions to concave ones. Therefore, if we color the surface according to the curvature in that direction (e.g., black in concave and white in convex), the surface's features will become crisp.

Figures 5, 6 show the results of our coloring. It can be seen that this method increases the color contrast on the features, thus enhancing them.

Formally, given a vertex with curvature $\kappa_{p}$ in the prominent direction, its color is defined as

$$
\text { color }=\tan ^{-1}\left(\lambda \kappa_{p}\right),
$$

where $\lambda$ is a parameter. Setting the value of $\lambda$ lets the user control the contrast between the convex and concave regions.

\subsubsection{Enhancement of the object's features}

Scanned archaeological objects are often noisy and unsuitable for further processing and visual analysis, either because of the erosion that they underwent during the years or due to scanning noise. We propose a new shape enhancement approach, which consists of two steps-bilateral filtering and inverse curvature flow - each makes use of our prominent field to guide the 

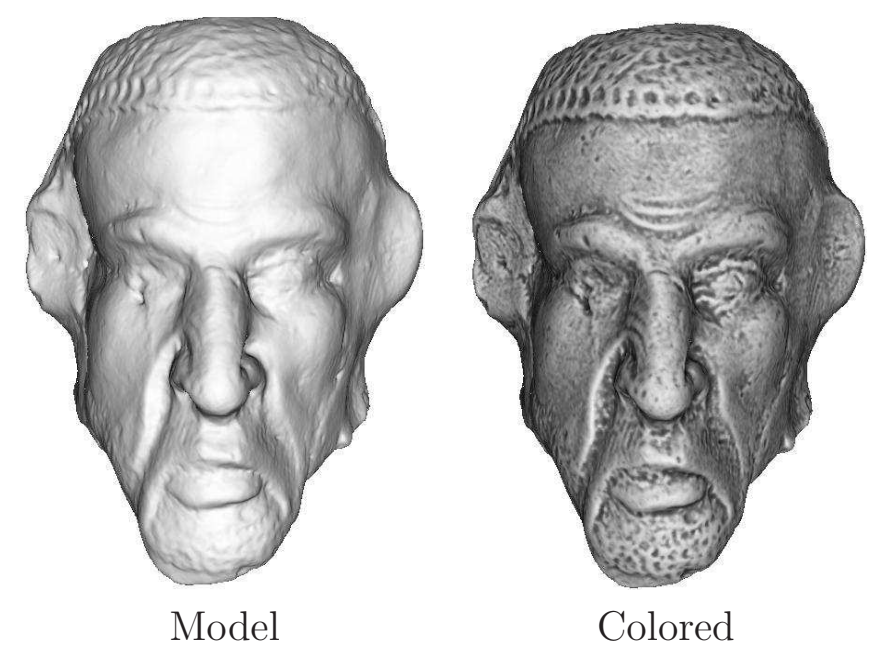

Figure 6: Coloring (a Persian-period figurine)

smoothing and enhancement directions.

Figure 7 illustrates some of our results. It can be seen that our enhancement indeed emphasizes the features and removes the noise from the original surface, making the visualized objects better suited for human analysis.

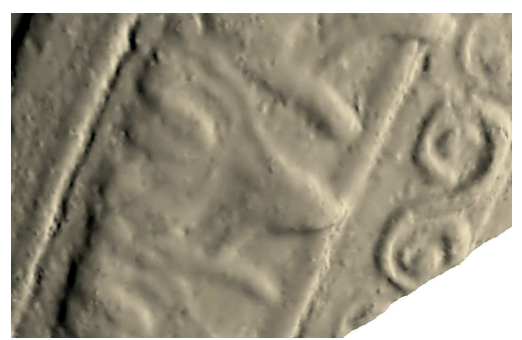

Model

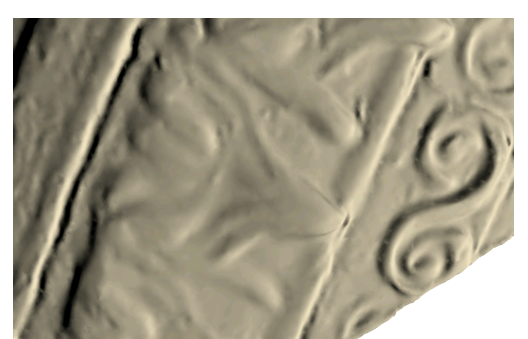

Enhancement

Figure 7: Enhancement (detail of a worn Hellenistic relief bowl)

Step 1 - Bilateral filtering: A bilateral filter sets the position of a vertex to a weighted average of its neighbors on the mesh (namely, the vertices connected by an edge to the vertex). The weights depend both on the distance between the vertices and on their similarity. In our case, when dealing with a point close to an edge, we would like to use in our computation only points that lie along the edge in order to remove the noise, and not points that traverse it, which would eliminate the 3D edge. We therefore propose 
to base the similarity component on the distance between the vertices along the prominent direction.

Formally, let $p$ be a point on the surface, $N(p)$ be the set of its neighbors, $d_{j}=\left\|p-p_{j}\right\|$ be the Euclidean distance between $p$ and $p_{j}$, and $n_{p}$ be the normal at $p$. In Fleishman et al. (2003) it is proposed to define the similarity as the distance between $p_{j}$ and $p$ 's tangent plane $h_{j}=\left\langle n_{p}, p-p_{j}\right\rangle$, so that smoothing is performed when $p_{j}$ is close to the tangent plane of $p$. In order to further prevent smoothing of edges, we propose to add to this definition a term that depends on $r_{j}$, the projection of $p-p_{j}$ along the prominent direction. This causes points flanking an edge to be considered distant (i.e., a high value for $r_{j}$ ). Thus, smoothing will not be performed across edges. This is done by multiplying the weights suggested in Fleishman et al. (2003) by the term $e^{-r_{j}^{2} / 2 \sigma_{p}^{2}}$. Hence, our similarity-based change of $p$ in its normal direction is

$$
\delta_{p}=C \sum_{j \in N(p)} e^{-d_{j}^{2} / 2 \sigma_{c}^{2}} \cdot e^{-h_{j}^{2} / 2 \sigma_{s}^{2}} \cdot e^{-r_{j}^{2} / 2 \sigma_{p}^{2}} \cdot h_{j}
$$

yielding a new position for $p^{\prime}=p+\delta_{p}$, where $C$ is the normalization coefficient.

In the implementation $\sigma_{s}=0.5 \sigma_{c}, \sigma_{p}=0.4 \sigma_{c}$, only $\sigma_{c}$ has to be supplied by the user, determining the amount of smoothing. It is common to slightly smooth the object prior to computing the distances.

Step 2 - Inverse-curvature flow: The inverse-curvature flow is a high frequency filter that updates the position of a vertex so as to increase the absolute value of its curvature. It can be based on the mean, maximum, minimum, or any other type of curvature. While the inverse-curvature flow manages to enhance features, it suffers from two drawbacks. First, it often creates spurious features on the surface, in addition to the enhanced features. This is so since for points in near-flat-regions with locally high curvature, values are inadvertently enhanced. Second, it is an iterative process that has no well-defined stopping criterion, causing unnaturally exaggerated features.

We propose a new inverse-curvature flow, which is based on two modifications to the standard flow. To solve the first problem, the curvature is computed in the prominent direction, enhancing only the real features. To solve the second problem, a new stopping criterion is suggested, which is based on the intuition in which a point should not exceed the extremum of the height function in the neighborhood of the point. 


\subsubsection{Axially-symmetric vessels/fragments with 3D designs}

These items constitute a sub-set of objects-in-relief, as they require both a clear representation of their designs as described above and a correct placing/alignment of the vessel/fragment in 3D space, for the drawing. This is made possible by combining our algorithm with the algorithm developed by Karasik and Smilansky (2008), which inter alia, calculates automatically the rotational axis of the potsherds/vessels and consequently places the fragments of axially-symmetric vessels in their correct angle.

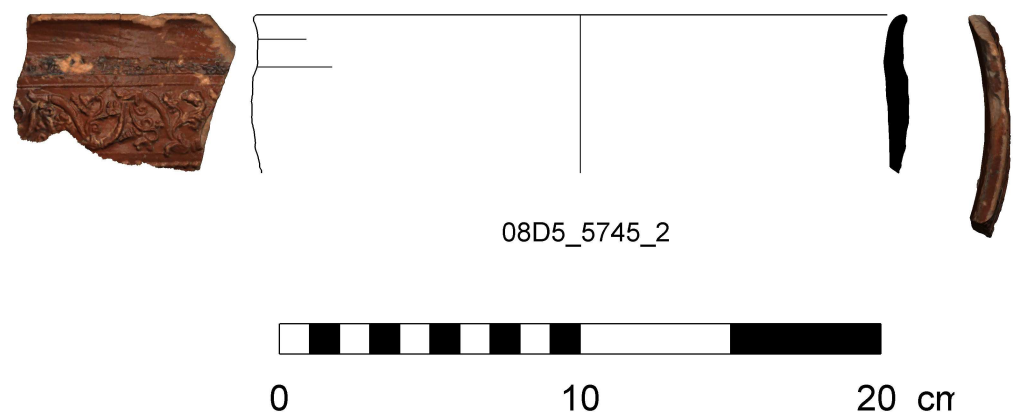

(a) (Karasik and Smilansky, 2008)

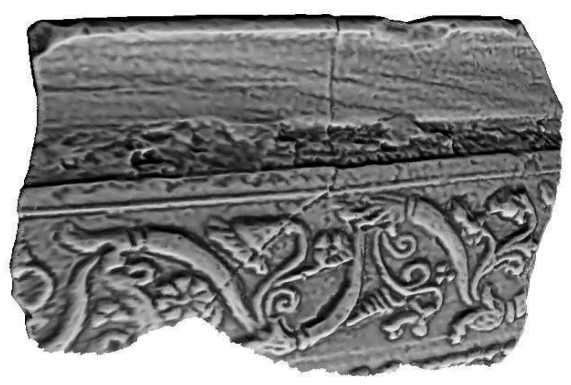

(b) D.o.R system

Figure 8: Positioning of Hellenistic relief bowl: (a) Result obtained by Karasik and Smilansky (2008); (b) Results obtained by our algorithm, where the object is automatically placed at the same orientation.

Their algorithm defines the concept of "optimal positioning" quantitatively. Given an axially symmetric body, all the points which are at the same height $h$ along the axis of symmetry are at the same distance $\rho$ from the axis. Plotting $h$ vs. $\rho$ for all the points on the surface, one gets a line that is the profile of the vessel. This operation projects the points of the vessel to a single plane - the reference plane. To prevent a case where the assumed 
axis is not the true axis, they find the axis in which all axially-symmetric points fall on the same point on the curve.

To combine our work with (Karasik and Smilansky, 2008), we run their code and use the returned axis to optimally position the object before applying our technique. Fig. 8 shows a fragment of a relief bowl - a common Hellenistic shape, where in (a) the result of Karasik and Smilansky (2008) is presented and in (b) the axis they computed is utilized by our system.

\subsection{Relief Extraction}

For certain objects it is useful to visualize only the outline of the design. This is true in cases where the outline encapsulates the pertinent information, such as the seal in Fig. 9 or the stamped handle in Fig. 10. To this end, we developed an algorithm that extracts the outline of the relief from its background (base). Mathematically, a relief on a surface is a part which is
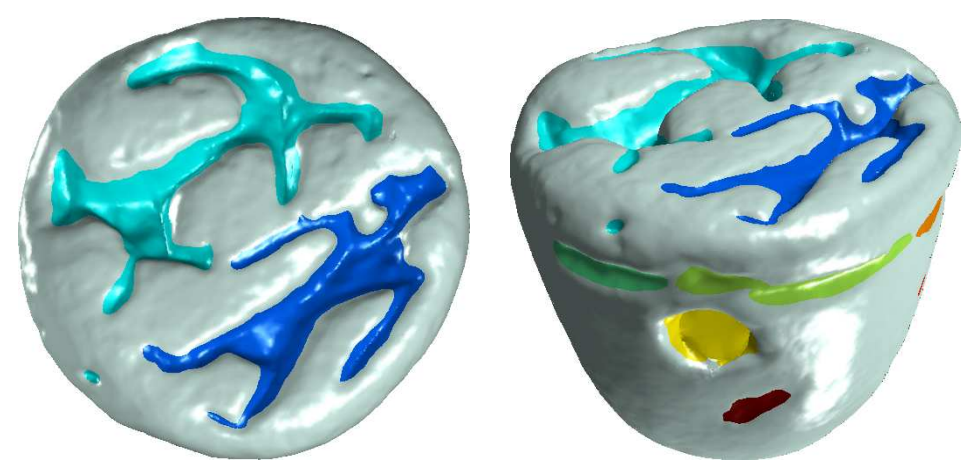

Figure 9: Relief Extraction (Iron Age seal)

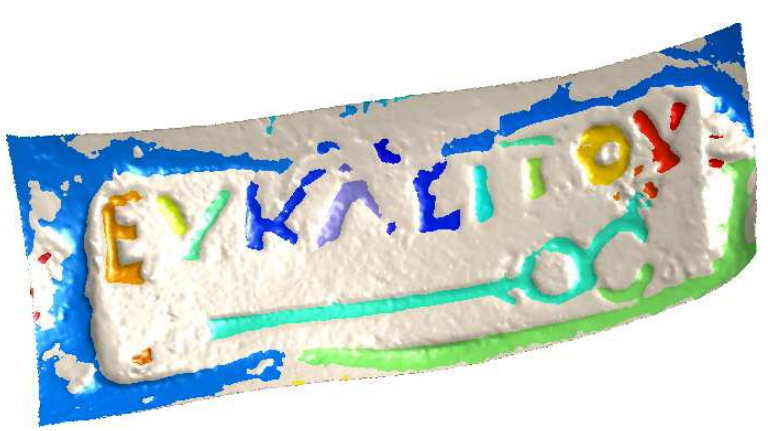

Figure 10: Relief Extraction (Hellenistic stamped handle) 
above some base (or below in case of an embossed relief). This means that we can identify reliefs by measuring heights on a surface. However, contrary to a height function defined on a 2D planar-base domain (photographs for example), the boundaries of 3D objects are curved surfaces and there is no clear base for measuring the relative height of points.

Towards this end, we consider the boundary surface of a 3D reliefed object as a composition of two components: a base surface and a height, which is a scalar function defined over the base in the normal direction to the base surface. Our only assumption is that the mean curvature of the base surface is smaller or equal to the curvature of the actual surface. If we could find an appropriate base surface, we would be able to measure the height of each surface point and use a threshold to define reliefs. However, given a boundary representation of an object, the decoupling of these components is unknown.

Our key observation is that there is no need to extract the real base surface to estimate details (Zatzarinni et al., 2009). The height function itself contains all the required information to separate the relief from the base. Hence, we only need a good estimation of the height and not the base surface itself. Interestingly, this turns out to be easier. We show that to measure height we only need an estimation of the normals of the base surface, and not the surface itself. Based on the base normals, we can define relative height differences between all the points on the model. By solving a global optimization problem we eventually reach a height definition for all points. The distribution of the height values are modeled as two Gaussian (Normal) distributions (i.e. a Gaussian mixture model (GMM)) (McLachlan and Basford, 1988). The reliefs are extracted by thresholding the height function, such that the two Gaussian distributions are separated, where the relief points belong to one distribution and the base points to the other.

Our basic algorithm consists of three parts. First, the normals $\overrightarrow{n_{B}}$ of the base $B$ are estimated using an adaptive Gaussian filtering on the surface. Since the surface can have different feature sizes in different areas, the challenge is to find the correct degree of smoothing for every normal, i.e. the standard deviation $\sigma$ of the Gaussian filter. If the smoothing is too strong, the normals of the base will be deformed. If the smoothing is too weak, the normals will fit the original surface and not the base. Therefore, $\sigma$ should be adapted locally to the surface. We perform the estimation in a manner similar to Kolomenkin et al. (2009).

Second, the estimated normals are used in a global minimization problem to find the height $h$ of every vertex. Locally, the height difference between 
adjacent points is computed using their base normals. These differences are then integrated to yield a consistent height map of the surface.

Third, once the height of every vertex is determined, we aim to segment the surface into two disjoint components: background and relief. This is performed using a threshold on the height function. The relief is defined as the union of all the points that are higher than this threshold above the base. The threshold is found by examining the histogram of the height values and approximating it with a Gaussian mixture model with two Gaussians.

\section{User interface}

The beta version of the system that implements the algorithm for computing 3D curves and coloring has been released (D.o.R System, 2012). It can be used as an interactive computerized tool that allows its operator to control the algorithm's parameters. This notwithstanding, all these manipulations are constrained by the 3D shape of the artifact and the results of the algorithms applied to it.

The interface presented to the user is shown in Figure 11. After selecting a 3D model from some directory, the user is presented with the object (Figure 11(a)). Since this is a three dimensional model, the user can manipulate it by rotating and translating it with the mouse. In addition, the user can change the lighting directions by manipulating the sphere-like icon.

Then, the user decides how to visualize the object, by selecting a sub-set of options from a menu. In particular, the user can choose to draw ridges, valleys, and relief curves, each in a different color. In this case, the algorithm described in Section 3.2.2 is applied. The user can also choose to color the object, as illustrated in Figure 11(b). In this case, the algorithm applied is the one described in Section 3.2.3. As can be seen for example in Figure 11, in the colored version it is much easier to read both the cuneiform letters and the stamps.

Once the desirable visualization has been selected, the user can save it in two manners. The first is an image, which can be incorporated into archaeological reports etc. The second is a 3D object (such as in a VRML format), which can be displayed by any available $3 \mathrm{D}$ viewer.

All the operations described are performed in interactive times. For example, loading a rather complex model consisting of 300,000 triangles, may take a couple of seconds. The other operations are instantaneous. 


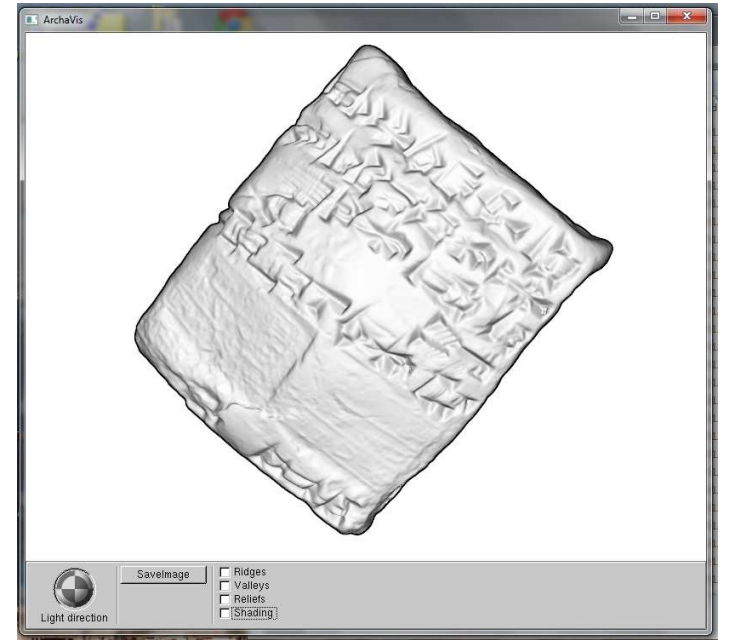

(a) The input model

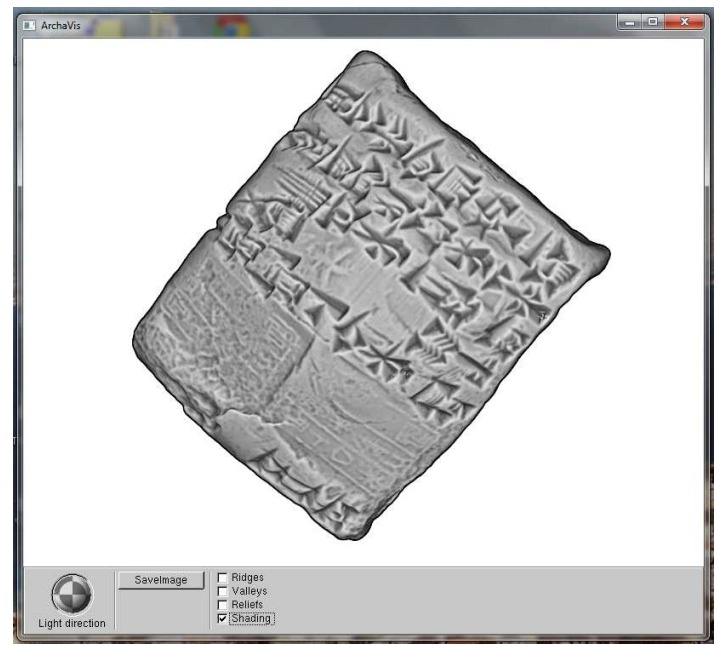

(b) After applying coloring

Figure 11: The screen presented to the user (Sumerian administrative tablet)

In the future, we intend to improve the user interface, taking into account feedback from archaeologists.

\section{Summary and Outlook}

Regarding several categories of complex archaeological objects and designs, the protocols described here have addressed and to a large extent solved the problems emanating from other recording and visualization methods. Dozens of objects with relief designs and objects-in-the-round can be scanned per day efficiently and accurately, their 3D models preserving the entire information about the objects' shape and designs. This practice, as mentioned in our introduction, is already quite widespread in archaeological circles, and will further spread as 3D scanners become increasingly affordable. One concomitant result will be that current constraints on the number of objects published per report/catalogue and the number of views produced per object will practically disappear. Our methods were designed to build on these models in order to produce illustrations that allow speedy and accurate visual recognition of the distinctive features of shapes and designs. As mentioned, archaeologists' intuitive preference of contour drawings over surface rendering is accounted for by the domain of perceptual psychology.

Our methods of illustration thus mimic the "good old manual drawings," 
but they are accurate and practically costless. Since the scans preserve the entire morphological information digitally, users can produce any number of views/'drawings' from any given model with the push of the button. They can also choose the preferred visualization method, or any combination of methods (such as line drawings combined with scans, enhancement with coloring, line drawings with coloring, and so forth). All these manipulations, however, are constrained by the 3D shape of the artifact and the results of the algorithms applied to it. The illustrations in this paper demonstrate that in most cases the coloring method produced the best results (combined with enhancement when needed).

For site reports etc. published on paper, these images can be transformed into print quality $2 \mathrm{D}$ illustrations. The advantage of these methods of visualization will also be retained when eventually site reports and other archaeological publications become electronic. They are especially critical regarding objects uncovered in large amounts, such as the oil lamps and relief bowls illustrated above, which need to be efficiently recorded, published and researched, the latter based inter alia on visually examining hundreds of comparanda. To provide one quantitative example: The Tel Dor excavations in Israel, co-directed by one of us (A. G.), uncovered between 2005-2010 fragments of about 500 Hellenistic and Roman lamps/lamp fragments with relief designs and about 500 fragments of Hellenistic relief bowls. Studying them will require comparisons with dozens of other corpora of 'similar' objects from other sites.

The Relief Extraction method provides a similar cost-effective means of visualizing objects in cases where only the contours of the designs are of interest.

The procedure described here, however, is only a first step in a process the end product of which should be an algorithm for computerized comparison and classification of such complex objects. As mentioned, this has to a large extent been achieved by Karasik and Smilansky (2011) for plain, axiallysymmetric pottery. The input to these procedures will be the curves and functions of the curves recovered by the algorithms described above. The functions will depend on geometric properties of the curves, such as curvatures, lengths, and the heights of the step edges. The relative importance of these parameters will be determined by the archaeologists who are the domain experts on the specific tasks. Eventually, then, when digital reports will also contain searchable databases of shape information, the process of searching for corollaries for one's object - a mandatory step for any study dealing 
with artifacts - would be reduced to querying multiple on-line databases according to morphological / textural criteria-pending the existence of the proper algorithms.

Such methods are also a prerequisite for addressing the ever growing complexity and sophistication of modern archaeological research. For example, questions such as the uniformity of 'similar' vessels/designs in order to differentiate between products of several workshops require the comparison of attributes that where hitherto impossible to comprehensively compare (visually). All these have important implications for issues such as technology, organization of production, and consequently social structure.

\section{Acknowledgements}

We acknowledge the close cooperation of Avshalom Karasik, Ilan Sharon, Uzy Smilansky, and Svetlana Matskevich of the Computerized Archaeology Laboratory at the Institute of Archaeology, Hebrew University, Jerusalem. We thank Andrea Berlin, Boston University; Adi Erlich, the University of Haifa; and Susan Rotroff, Washington University in St. Louis - experts on ceramics, figurines and other archaeological media - for discussing with us the methods presented here. We received important input from Noga Ze'evi. As a chief draftsperson for the Israel Antiquities Authority she has illustrated (manually....) thousands such artifacts. We thank the British Museum staff for the permission to illustrate the images in Fig. 1:3, 4 (C) The Trustees of the British Museum) and the directors of the Rheinisches Landesmuseum, Trier, for letting us reproduce the illustration in Fig. 1:2. The other illustrations are from the database of the Tel Dor Excavation Project in Israel, directed by Sharon and Gilboa. Figure. 1 was compiled by Anat Regev of the Zinman Institute of Archaeology, University of Haifa. The 3D scanner was purchased by the Zinman Institute in collaboration with Uzy Smilansky, the Weizmann Institute of Science, and supported by Israel Science Foundation Grant No. 727/05, granted to Gilboa, Daniel Nadel, and Ezra Marcus. Work was also supported by The Joint Technion-University of Haifa Research Fund.

\section{References}

Adkins, L., Adkins, R. A., 1989. Archaeological Illustration. Cambridge Manuals in Archaeology. Cambridge: University Press. 
Adler, K., Kampel, M., Kastler, R., Penz, M., Sablatnig, R., Schindler, K., Tosovic, S., 2001. Computer aided classification of ceramics - achievements and problems. In: Brner, W., Dollhofer, L. (Eds.), Proceedings of the 6th International Workshop on Archaeology and Computers. Vienna, Austria, pp. $3-12$.

Aliaga, D., Law, A., Yeung, Y., 2008. A virtual restoration stage for realworld objects. ACM Transactions on Graphics 27, 149:1-10.

Bailey, D., 1996. A Catalogue of Lamps in the British Museum IV: Lamps of metal and Stone and Lampstands. British Museum, London.

Biederman, I., 1995. Chapter 4: Visual object recognition. In: Kosslyn, S.M., Osherson, D.N. (Eds.), Visual Cognition. MIT Press, Cambridge, MA and London, pp. 121-165.

Biederman, I., Ju, G., 1988. Surface vs. edge-based determinants of visual recognition. Cognitive Psychology 20, 38-64.

Brutto, M., Spera, M., 2011. Image-based and range-based 3D modeling of archaeological cultural heritage: the Telamon of the temple of Olympian Zeus in Agrigento (Italy). In: International Archives of Photogrammetry, Remote Sensing and Spatial Information Sciences 38 (Part 5).

DeCarlo, D., Finkelstein, A., Rusinkiewicz, S., Santella, A., 2003. Suggestive contours for conveying shape. ACM Transactions on Graphics 22, 848-855.

DeCarlo, D., Rusinkiewicz, S., 2007. Highlight lines for conveying shape. In: Proceedings of the 5th International Symposium on Non-photorealistic Animation and Rendering. pp. 63-70.

Dillon, B. D., 1992. The Student's Guide to Archaeological Illustrating. Archaeological Research Tools 1. Los Angeles: University of California.

Do Carmo, M. P., 1976. Differential geometry of curves and surfaces. Prentice-Hall.

D.o.R System, 2012. http://webee.technion.ac.il/labs/cgm/ComputerGraphics-Multimedia/Software/EdgeDetection/EdgeDetection.html.

Eigensatz, M., Sumner, R., Pauly, M., 2008. Curvature-domain shape processing. Computer Graphics Forum 27, 241-250. 
Fleishman, S., Drori, I., Cohen-Or, D., 2003. Bilateral mesh denoising. ACM Transactions on Graphics 22, 950-953.

Gassner, V., 1997. Das Südtor der Tetragonos-Agora: Keramik und Kleinfunde. Forschungen in Ephesos 13.1.1. Austrian Academy of Sciences, Vienna.

Gilboa, A., Karasik, A., Sharon, I., Smilansky, U., 2004. Towards computerized typology and classification of ceramics. Journal of Archaeological Science 31, 681-694.

Goethert-Polaschek, K., 1985. Katalog der römischen Lampen des Rheinischen Landesmuseums Trier. Bildlampen und Sonderformen. Trierer Grabungen und Forschungen 15. Von Zabern, Mainz.

Gooch, B., Gooch, A., 2001. Non-Photorealistic Rendering. Peters Ltd., Natick, MA.

Gooding, D. C., 2008. Envisioning explanation: the art in science. In: Frischer, B., Dakouri-Hild, A. (Eds.), Beyond illustration: 2D and 3D digital technologies as tools for discovery in archaeology. BAR international series 1805. Archaeopress, Oxford, pp. 1-19.

Griffiths, N., Jenner, A., Wilson, C., 1990. Drawing Archaeological Finds. Occasional paper No. 13, Institute of Archaeology, University College. Archetype, London.

Grosman, L., Smikt, O., Smilansky, U., 2008. On the application of 3-D scanning technology for the documentation and typology of lithic artifacts. Journal of Archaeological Science 35, 3101-3110.

Hanke, K., Moser, M., Grimm-Pitzinger, A., Goldenberg, G., Toechterle, U., 2008. Enhanced potential for the analysis of archaeological finds based on 3D modeling. In: The International Archives of the Photogrammetry, Remote Sensing and Spatial Information Sciences XXXVII, Part B5. pp. $187-192$.

Hörr, C., Brunner, D., Brunnett, G., 2007. Feature extraction on axiallysymmetric pottery for hierarchical classification. Computer-Aided Design and Applications 4, 375-384. 
Howland, R., 1958. Greek Lamps and their Survivals. The Athenian Agora IV. American School of Classical Studies at Athens, Princeton.

Joukowsky, M., 1986. A Complete Manual of Field Archaeology: Tools and Techniques of Field Work for Archaeologists. Prentice-Hall, New York.

Judd, T., Durand, F., Adelson, E., 2007. Apparent ridges for line drawing. ACM Transactions on Graphics 22, 19:1-7.

Kampel, M., Sablatnig, R., 2007. Rule based system for archaeological pottery classification. Pattern Recognition Letters 28, 740-747.

Karasik, A., Smilansky, U., 2008. 3D scanning technology as a standard archaeological tool for pottery analysis: practice and theory. Journal of Archaeological Science 35, 1148-1168.

Karasik, A., Smilansky, U., 2011. Computerized morphological classification of ceramics. Journal of Archaeological Science 38, 2644-2657.

Kindlmann, G., Whitaker, R., Tasdizen, T., Moller, T., 2003. Curvaturebased transfer functions for direct volume rendering: methods and applications. In: IEEE Visualization. pp. 67-76.

Koenderink, J., 1984. What does the occluding contour tell us about solid shape? Perception 13, 321-330.

Kolomenkin, M., Shimshoni, I. Tal, A., 2008. Demarcating curves for shape illustration. ACM Transactions on Graphics 27, 157:1-9.

Kolomenkin, M., Shimshoni, I., Tal, A., 2009. On edge detection on surfaces. In: IEEE Conference on Computer Vision and Pattern Recognition (CVPR). pp. 2767-2774.

Kolomenkin, M., Shimshoni, I., Tal, A., 2011. Prominent field for shape processing and analysis of archaeological artifacts. International Journal of Computer Vision 94, 89-100.

Levoy, M., Rusinkiewicz, S., Curless, B., Ginzton, M., Ginsberg, J., Pulli, K., Koller, D., Anderson, S., Shade, J., Pereira, L., Davis, J., Fulk, D., 2000. The digital michelangelo project: 3D scanning of large statues. In: ACM SIGGRAPH 2000. pp. 131-144. 
Levy, T., Petrovic, V., Wypych, T., Gidding, A., Knabb, K., Hernandez, D., Smith, N., Schlulz, J., Savage, S., Kuester, F., Ben-Yosef, E., Buitenhuys, C., Barrett, C., Najjar, M., DeFanti, T., 2010. On-site digital archaeology 3.0 and cyber-archaeology: into the future of the past - new developments, delivery and the creation of a data avalanche. In: Forte, F. (Ed.), CyberArchaeology. BAR International Series 2177. Archaeopress, Oxford, pp. $135-153$.

Li, J.-B., Li, M., Gao, H., 2011. Automatic 3D digital patching for fragmented cultural relics repair. Assembly Automation 31, 224-231.

Luo, T., Li, R., Zha, H., 2011. 3D line drawing for archaeological illustration. International Journal of Computer Vision 94, 23-35.

Mandel, U., 1988. Kleinasiatische Reliefkeramik der mittleren Kaiserzeit. Pergamenische Forschungen 5. W. de Gruyter, Berlin and New York.

Mara, H., Krömker, S., Jakob, S., Breuckmann, B., 2010. GigaMesh and Gilgamesh: 3D multiscale integral invariant cuneiform character extraction. In: VAST: International Symposium on Virtual Reality, Archaeology and Cultural Heritage. pp. 131-138.

Mara, H., Sablatnig, R., 2005. Semiautomatic and automatic profile generation for archaeological fragments. In: Proc. of the 10th Computer Vision Winter Workshop. pp. 123-134.

Martínez Carrillo, A., Rubio Paramio, M., Navas Lara, J., 2008. 3D data capture and visualization of archaeological pottery. In: Ioannides, M., Addison, A., Georgopoulos, A., Kalisperis, L. (Eds.), The 14th International Conference on Virtual Systems and Multimedia VSMM. pp. 20-25.

McLachlan, G., Basford, K., 1988. Mixture Models: inference and applications to clustering. Statistics: Textbooks and Monographs. Dekker, New York.

Mlynarczyk, J., 1997. Alexandrian and Alexandria-Influenced Mould-Made Lamps of the Hellenistic Period. BAR International Series 677. Archaeopress, Oxford.

Ohtake, Y., Belyaev, A., Seidel, H., 2004. Ridge-valley lines on meshes via implicit surface fitting. ACM Transactions on Graphics 23 (3), 609-612. 
Orton, C., Tyres, P., Vince, A., 1993. Pottery in Archaeology. University. Press, Cambridge.

Perlzweig, J., 1961. Athenian Agora VII: Lamps of the Roman Period. American School of Classical Studies at Athens, Princeton.

Pires, H., Ortiz, P., Marques, P., Sanchez, H., 2006. Close-range laser scanning applied to archaeological artifacts documentation. virtual reconstruction of an XVIth century ceramic pot. In: VAST: International Symposium on Virtual Reality, Archaeology and Cultural Heritage. pp. 284-289.

Pobelome, J., van den Brandt, J. Michiels, B., Evsever, G., Degeest, R., Waelkens, M., 1997. Manual drawing versus automated recording of ceramics. In: Waelkens, M., Pobelome, J. (Eds.), Sagalassos IV. Acta Archaeologica Lovaniensia Monographiae 9. Leuven, pp. 533-538.

PRISM, 1997. http://prism.engineering.asu.edu/research/3dk.php.

Rosenthal-Heginbottom, R., 1995. Imported Hellenistic and Roman pottery. In: Stern, E., Berg, J., Gilboa, A., Guz-Zilberstein, B., Raban, A., Rosenthal-Heginbottom, R., Sharon, I. (Eds.), Excavations at Dor, Final Report. Areas A and C: The Finds. Qedem Reports 2, vol. IB. Hebrew University, Jerusalem, pp. 183-288.

Rotroff, S., 1982. The Athenian Agora XXII: Athenian and Imported Mouldmade Bowls. American School of Classical Studies at Athens. Princeton.

Rusinkiewicz, S., Burns, M., DeCarlo, D., 2006. Exaggerated shading for depicting shape and detail. ACM Transactions on Graphics 25, 1199-1205.

S.H.A.P.E, 2002. http://www.lems.brown.edu/shape/.

Simon, A., Van Alfen, D., Razdan, A., Farin, G., Bae, M., Rowe, J., 2002. 3D modeling for analysis and archiving of ceramic vessel morphology: A case study from the American Southwest. In: Kars, H., Burke, E. (Eds.), Proceedings of the 33rd International Symposium on Archaeometry. Geoarchaeological and Bioarchaeological Studies, Vrije Universiteit, Amsterdam. pp. 257-263.

Steiner, M., 2006. Approaches to Archaeological Illustration. Council for British Archaeology, York. 
Toler-Franklin, C., Brown, B., Weyrich, T., Funkhouser, T., Rusinkiewicz, S., 2010. Multi-feature matching of fresco fragments. ACM Transactions on Graphics 29, 185:1-12.

Van Gool, L., Pollefeys, M., Proesmans, M., Zalesny, A., 2000. The MURALE project: image-based 3d modeling for archaeology. In: Niccolucci, F. (Ed.), VAST: International Symposium on Virtual Reality, Archaeology and Cultural Heritage. pp. 53-63.

Virtual Hampson, 2010. http://cast.uark.edu/home/research/archaeologyand-historic-preservation/archaeological-geomatics/archaeological-laserscanning/hampson-museum.html.

Yoshizawa, S., Belyaev, A., Seidel, H., 2006. Smoothing by example: mesh denoising by averaging with similarity-based weights. In: IEEE International Conference on Shape Modeling and Applications. Los Alamitos, CA., pp. 9-19.

Zatzarinni, R., Tal, A., Shamir, A., 2009. Relief analysis and extraction. ACM Transactions on Graphics 28, 136:1-9. 